\title{
ESTRATEGIA PARA EL APROVECHAMIENTO DE UN ENTORNO VIRTUAL EN EL APRENDIZAJE DE UNA SEGUNDA LENGUA
}

\author{
STRATEGIES FOR THE USE OF A VIRTUAL ENVIRONMENT IN \\ SECOND LANGUAGE LEARNING
}

\author{
Iliana Real Poveda, MAE \\ Docente UNEMI \\ ilianarealpovea@gmail.com \\ Yonaiker Navas Montes, Ph.D \\ Prometeo Senescyt
}

\section{RESUMEN}

La Organización de las Naciones Unidas para la Educación, la Ciencia y la Cultura (UNESCO), desde 1948, destaca la importancia del aprendizaje de una segunda lengua debido a las potencialidades que supone a nivel profesional, académico, social, cultural e incluso personal. Entendiendo que se avanza a una sociedad tecnológica, los procesos de enseñanza y aprendizaje rompen los esquemas tradicionales e incorporan entornos virtuales de aprendizaje que permiten la aplicación de tecnologías. Al aprender una lengua extranjera, se tiende a "traspasar" de alguna manera el conocimiento sobre las estructuras y rasgos de la lengua materna. A esta influencia de la lengua materna sobre la lengua extranjera se llama "transferencia" que en muchas ocasiones obstaculiza la producción en esta segunda lengua. El propósito de ésta investigación es describir una estrategia empleada para el aprovechamiento del entorno virtual en el aprendizaje de los módulos de inglés con el uso de la plataforma Moodle para los 114 estudiantes de Ciencias de la Salud y Ciencias de la Ingeniería, considerados parte de la generación " $Y$ ", a través de un estudio de caso único, factual e intrínseco, teniendo un nivel descriptivo, heurístico e inductivo. Los resultados de la investigación determinaron que con el uso del entorno virtual a través de una estrategia el aprendizaje de una segunda lengua presenta mayores oportunidades ya que docente y alumnos pueden estar separados con respecto a la sincronía y esto no es obstáculo para que el aprendizaje se lleve a cabo con éxito, esta herramienta de apoyo ayuda a sellar el conocimiento adquirido para luego perfeccionarlo con la práctica.

Palabras clave: Entorno virtual de aprendizaje, Inglés, Proceso de enseñanza-aprendizaje, TIC

\section{ABSTRACT}

The United Nations Educational, Scientific and Cultural Organization (UNESCO) has highlighted since 1948 the importance of learning a second language because of its professional, social, cultural, academic, and even personal potentials. Understanding that it moves towards a technological society, the processes of teaching and learning break the traditional schemes and incorporate new virtual learning environments that allow the implementation of technologies. By learning a foreign language, it tends to "pass" in somehow the knowledge about the structures and features of the mother tongue into the foreign language. This influence of the mother tongue on the foreign language is called "transfer" which often impedes production in the second language. The purpose of this research is to create a virtual learning environment in the English modules using Moodle for 114 UNEMI students of Health and Engineering Sciences, who are considered part of the generation " $Y$ " through a single, factual and intrinsic case study, with a descriptive, heuristic and inductive level. The results of this research determined that the use of ICT tools when learning a second language presents greater opportunities for the teacher and students because they both can be separated with respect to the synchrony and this is not an obstacle, so learning is conducted with success; this support tool helps the acquired knowledge to become perfect with practice.

Key words: Virtual Learning Environment, English, teaching-learning process, ICT 


\section{Introducción}

La Organización de las Naciones Unidas para la Educación, la Ciencia y la Cultura (UNESCO), ha liderado el derecho universal a la educación desde 1948, en este contexto la enseñanza de una segunda lengua constituye un elemento importante en la educación contemporánea debido a las potencialidades que supone para los individuos a nivel académico, laboral, cultural e incluso a nivel personal.

El desarrollo de una sociedad tecnológica reclama de sistemas que ofrezcan una educación más humanista, en la que se tome en cuenta al individuo que aprende, donde se propicie participación, reflexión y toma de conciencia de su propio aprendizaje como proceso holístico, integrador e integral, lo que presupone importantes transformaciones en los procesos de enseñanza y aprendizaje a todos los niveles, en especial para este caso estudio al nivel superior.

Es necesario que dicha transformación de los procesos de enseñanza y aprendizaje rompa con los esquemas tradicionales que aún predominan en la práctica académica, el estudiante no tiene que reproducir el material a estudiar de forma pasiva, el dicente tiene que ser un ente activo, comprometido e independiente, un verdadero emprendedor para luego convertirse en el líder que necesita el siglo XXI.

De acuerdo con la UNESCO, "la tecnología digital es una herramienta útil en el proceso de enseñanza aprendizaje, por cuanto convierte al estudiante en un ser creativo, reflexivo, analítico y productivo" (Orozco, 2011, p.25) destaca la importancia del uso de los recursos de las Tecnologías de la Información y la Comunicación (TIC) para motivar la práctica educativa, generar interés y estrategias tanto de enseñanza como de aprendizaje.

Existen corrientes internacionales que integran las tecnologías en los procesos de enseñanza y aprendizaje de lenguas extranjeras, estas son conocidas por sus siglas en inglés como CALL (Computer-Assisted Language Learning, Aprendizaje de lenguas asistido por computadoras) y TELL (Technology Enhanced Language Learning, Aprendizaje de lenguas impulsado por tecnologías), en ambos casos se reúnen grupos de investigadores y docentes los cuales estudian y llevan a cabo prácticas de integración de tecnologías en la enseñanza de otras lenguas.

En los años 90, CALL era la aplicación que realizaba las búsquedas y estudios de los sistemas de aprendizaje y enseñanza de las lenguas (Levy,
1997), por lo tanto incluía un amplio espectro de aplicaciones y enfoques basados en el uso de las TIC para el aprendizaje de una segunda lengua. De acuerdo con Orozco (2011, p.22) "las competencias docentes para su aplicación en la gerencia de aula deben estar ligadas a la comprensión de quiénes somos y hacia dónde queremos llegar" lo que se traduce en direccionar los objetivos personales hacia la institución, logrando canalizar energías y unir esfuerzos para definir compromisos concretos y medibles. Las autoras afirman que las etapas del proceso de enseñanza aprendizaje de una segunda lengua son: planificación, organización, ejecución y evaluación.

Al aprender una lengua extranjera (L2), se tiende a "traspasar" de alguna manera el conocimiento sobre las estructuras y rasgos de la lengua materna (L1) a esa L2. A esta influencia de la L1 sobre la L2 se llama "transferencia". Sin embargo, con frecuencia esa transferencia provoca errores; en este caso, a esa "transferencia de efectos negativos" se la denomina "interferencia" (Valenzuela, 2002). Cabe destacar por ejemplo que el español e inglés son lenguas que tienen su origen del Proto-Indo-Europeo, por consiguiente hay muchos aspectos que ambas lenguas comparten especialmente a nivel gramatical (morfológico y sintáctico). Además hay similitudes léxicas porque un $40 \%$ del léxico de la lengua inglesa proviene del idioma francés que al igual que el español son lenguas latinas. Ahora bien, hay una diferencia significativa entre el inglés y el español y es que la lengua inglesa es una lengua germánica, cuyas familias son el alemán, el sueco, el danés mientras que la lengua española está emparentada con el francés, el portugués, el italiano, el catalán que son lenguas romance, por consiguiente las diferencias o contraste entre el inglés y español serán evidentes en determinadas situaciones.

El aprendizaje de una segunda lengua requiere el dominio de los aspectos generales de la comunicación oral en la lengua materna, estos son:

1. Fonológico: Es el nivel donde más claramente se puede observar la interferencia de la L1 sobre la L2 (Valenzuela, 2002). Al respecto, los sistemas fonológicos de las lenguas tienen tres componentes: su lista de fonemas, el sistema que especifica cómo se actualizan estos fonemas en el habla real, es decir, cómo afecta el contexto real a la pronunciación concreta de cada fonema, son las reglas alofónicas (un alófono es la realización concreta y real de un fonema). Por último, el tercer componente es el que reúne las reglas de combinación de fonemas que se permiten o prohíben en cada lengua. Las reglas fonológicas difieren en los distintos idiomas por tanto encontramos dife- 
rencias notorias entre el inglés y el español. Estas diferencias entre las reglas fonológicas de la lengua inglesa y la española tienen mucho que ver en lo que se conoce como "acento extranjero", en otras palabras, un hispanoparlante hablando inglés con el acento de su lengua materna al igual que un angloparlante hablando español con acento inglés ambos están asimilando los fonemas que no existen en sus sistemas al más próximo de su L1 y además están pronunciando los sonidos de la L2 aplicando las reglas fonológicas de su lengua materna.

2. Morfológico: De igual forma que el aspecto anterior, el morfológico contiene diferencias importantes. La morfología se clasifica o divide en: flexiva y derivacional. La morfología flexiva se ocupa de los cambios en las palabras que la modifican para ajustarla a un contexto sintáctico dado (Valenzuela, 2002). En términos generales hay pocos morfemas flexivos y se distinguen porque al conectarse a una raíz no modifica la categoría sintáctica de la misma. Las interpretaciones que aportan los morfemas flexivos corresponden al número, género, posesión, afecto, tiempo verbal, entre otros. En la morfología derivativa los morfemas transforman la categoría sintáctica de la raíz a la que se enlazan o hay un cambio importante en su significado, por ejemplo podemos convertir un sustantivo, verbo o adjetivo en otra categoría (jugar - juego) (igual - igualdad). Otro aspecto a tratar en este tema son los "morfemas apreciativos" que son parte de la lengua española porque el diminutivo escasamente se lo encuentra en inglés, mientras que el aumentativo y peyorativo son ausentes totales en la lengua inglesa.

3. Semántico: Las diferencias léxico-semánticas entre dos idiomas causan graves confusiones y pueden incluso hacer que quien aprende el idioma lo vea "difícil" puesto que en muchos casos es imposible que haya una equivalencia exacta de una palabra, por ejemplo la palabra inglesa "spell" que es un verbo y sustantivo en español se traduce a deletrear como verbo y hechizo como sustantivo. Si analizamos los dos significados no tienen ninguna conexión y si no hay mayor conocimiento de vocabulario, la confusión es predecible.

4. Sintáctico: En ambas lenguas española e inglesa las categorías a nivel sintáctico son muy parecidas, sin embargo existen diferencias que citar:

- Diferencias entre los sustantivos (contables y no contables, en inglés hay mayor cantidad de sustantivos no contables siendo lo opuesto en español)

- Diferencias entre los adjetivos (en inglés obligatoriamente se escribe el adjetivo delante del sustantivo.

- Diferencias entre los artículos (hay seis formas que pueden ocasionar confusión: Artículo cero inglés por artículo indeterminado español, artículo cero inglés por artículo determinado español, artículo indeterminado inglés por artículo cero español, artículo indeterminado inglés por artículo determinado español, artículo determinado inglés por artículo cero español, y artículo determinado inglés por artículo indeterminado español).

- Presencia/ausencia del sujeto (en inglés es obligatorio siempre escribir o nombrar al sujeto, en español se incluye solamente para hacer contraste, dar énfasis sin que su ausencia afecte la comprensión de lo que se quiere expresar)

- Orden sujeto + verbo (en inglés esta regla tiene que cumplirse siempre, en español se puede colocar el sujeto en otros lugares de la oración sin afectar su significado debido a lo que aporta la información morfológica del verbo)

- Separación verbo + objeto (en ambos idiomas el orden básico es $\mathrm{S}+\mathrm{V}+\mathrm{O}$, en inglés esto se acata de manera más estricta así como se mencionó anteriormente en el caso del sujeto. No es válido de forma sencilla incluir otro constituyente entre el verbo y el objeto.

- La Voz Pasiva: En ambas lenguas, inglesa y española, la voz pasiva se construye de la misma forma, sin embargo en inglés es usada con más frecuencia debido a que en la voz pasiva se destaca el objeto directo porque toma la posición del sujeto y como resultado es el centro de atención. Además, la voz pasiva permite no dar protagonismo al sujeto cuando no se conoce el agente de una acción y más bien se desea enfocarse en la acción misma.

Al leer los párrafos anteriores, es fácil entender porqué a los jóvenes universitarios se les dificulta el aprendizaje de una segunda lengua, en tal sentido se hace necesario indagar en las características de este estudiante y las relaciones de éstas con su aprendizaje. Las características de los jóvenes universitarios son descritas por Córica y Dinerstein (2009), una de ellas es que son conocidos como generación " $Y$ " o "Net", adicionalmente se pueden mencionar las siguientes:

- Nacimiento: son los nacidos en los años 80 hasta el 2000.

- Valores: la inmediatez, libertad absoluta, relatividad en todo y aporte a grandes causas.

- Familia: informal, con rechazo al concepto 
de matrimonio para toda la vida donde divorciarse implica separarse del cónyuge y no de los hijos.

- Rol de géneros: se presenta una tendencia hacia la maternidad en edad avanzada.

- Educación: la escuela compite con internet, la televisión y los videojuegos.

- Trabajo: debe ser divertido para que los atraiga.

- Tecnología utilizada: lectura hipertextual, radio, teléfono fijo, cine, tv, computadora, laptop, internet, $\mathrm{mp} 3, \mathrm{mp} 4$, celular, GPS.

Conociendo estas características se puede hacer uso de las tecnologías para hacer atractivo el aprendizaje de una segunda lengua en el contexto universitario. En tal sentido, la universidad ha creado un entorno virtual de aprendizaje ya que el desarrollo de las aulas virtuales bajo software libre es una tendencia en el Ecuador y específicamente en la Universidad Estatal de Milagro (UNE$\mathrm{MI})$ se ha implementado Moodle como plataforma para la construcción de las mismas.

Estos entornos virtuales se adecuan a las necesidades y características de los universitarios, potencia el trabajo colaborativo, desarrollan pensamiento crítico y facilita el aprendizaje, por lo cual su aprovechamiento es beneficioso cuando dichos jóvenes desean aprender una segunda lengua. Es importante destacar que el trabajo colaborativo permite que cada estudiante sea responsable del aprendizaje, ya que según Calzadilla (2006), es el conjunto de capacidades, procedimientos, hábitos, valores y actitudes por parte de los alumnos que participan desarrollando relaciones interpersonales por medio del valor de la cooperación, creando de manera positiva empatía, tolerancia, amistad y confianza.

\section{Objetivos}

El objetivo general de esta investigación es describir la estrategia empleada para el aprovechamiento de un entorno virtual en el aprendizaje de los módulos de Inglés en los estudiantes de Ciencias de la Salud y Ciencias de la Ingeniería basados en la plataforma educativa Moodle de la UNEMI. Para el desarrollo del mismo se definieron como objetivos específicos en la investigación a) Fundamentar teóricamente el aprendizaje de una segunda lengua en los siguientes aspectos: fonológico, morfológico, semántico y sintáctico; b) Caracterizar a la generación " $Y$ " de la Universidad Estatal de Milagro; c) Describir la estrategia empleada para el aprovechamiento de un entorno virtual en el aprendizaje de los módulos de Inglés, basados en la plataforma educativa Moodle de la UNEMI.

\section{Materiales y métodos}

La investigación se realizó como un estudio de caso único definido por Denny (1978, p.370) como "un examen completo e intenso de una faceta, una cuestión o quizás los acontecimientos que tienen lugar en un marco geográfico a lo largo del tiempo", para esta investigación convergerán, de igual manera los planteamientos de Patton (1980) quien lo considera como una forma particular de recoger, organizar y analizar los datos.

Es particularista pues el estudio se centra en las actividades desarrolladas en los cuatro módulos de inglés narrados en la tabla 1 , teniendo un nivel descriptivo, heurístico e inductivo. El propósito de ésta investigación es la colaboración del docente hacia los estudiantes para construir un entorno virtual de aprendizaje en los módulos de inglés con el uso de la plataforma Moodle. También es de tipo factual según Guba y Lincoln (1981, p.374) e intrínseco (Stake, 1994) pues pretende alcanzar una mejor comprensión de las estrategias de aprendizaje empleadas por los estudiantes. Se planteó desde la modalidad observacional ya que es la principal técnica de recogida de datos.

Para realizar la estrategia se realizó una revisión bibliográfica sobre las implicancias del uso de las tecnologías en los jóvenes, poniendo en evidencia la necesidad de ajustarse en el abordaje de los diferentes aspectos que estas abarcan, entre ellos la apropiación de la misma tecnología, la mediación en el aprendizaje, entendido éste como una plataforma donde se construirán, deconstruirán y reconstruirán los mismos. Cuando la relación de las tecnologías se hace a través de entornos virtuales creados para fomentar el aprendizaje y éste se media por una estrategia que aprovecha los múltiples usos que ésta posee, es probable que el objetivo planteado en el aprendizaje resulte más exitoso ya que se apoya en el conocimiento sobre el conjunto dona de saberes previos con los que cuenta el estudiante y también sobre las actitudes con las cuales los sujetos enfrentan las tecnologías y afrontan los procesos de aprendizaje.

El aprendizaje en los estudiantes universitarios supone el desarrollo de capacidades que permiten, disponiendo de tecnologías para completar la formación, discutir, intercambiar, debatir conceptos, ideas e interpretaciones, favoreciendo el mismo en una segunda lengua. Diversos autores sostienen la pertinencia para asimilar nuevas visiones de la realidad Tunnermann, (1996; 2000), y la posibilidad de fundar la alfabetización científico tecnológica tan impulsada en los proyectos de la UNESCO (UNESCO, 1998). 
En la literatura pedagógica contemporánea se plantea con claridad el papel innovador que le corresponde a la educación superior y en particular a la universidad frente a los cambios sucesivos del entorno y la capacidad que desarrollan para adelantarse a los mismos, sobre todo en los jóvenes de la generación " $Y$ ".

Se recoge en la literatura pedagógica con claridad y evidencia el papel innovador de la Educación Superior en la sociedad y en este contexto la importancia del aprendizaje de una segunda lengua como lo es el inglés. Se plantea que la Universidad debe adecuar sus actuaciones a los cambios del entorno. En el proceso de formación que se lleva a cabo lo fundamental es preparar al hombre para resolver los problemas contextuales de la sociedad donde éste vive y se desarrolla. Este proceso se erige a partir de una concepción didáctica la cual se mueve en un continuo debate dialéctico basado en el principio martiano que consiste en educar en la vida social, por la vida y para la vida.

En la actualidad, el problema principal del proceso docente educativo consiste en desarrollar los vínculos que cotidianamente se establecen entre la universidad y la sociedad. De esta manera sepuede plantear que el proceso de aprendizaje de una segunda lengua parte de las relaciones didácticas que se dan entre los sujetos que participan, en correspondencia con los componentes organizativos esenciales del mismo, y está dirigido de un modo sistémico en el plano educativo, desarrollador e instructivo a la solución de los problemas sociales, en particular al desafío que implica el aprendizaje de una segunda lengua a través del aprovechamiento del entorno virtual que para tal efecto ha creado la UNEMI.

Ello se logra mediante la apropiación que el estudiante hace de la cultura que ha acopiado la humanidad en su desarrollo, a través de su participación activa y consciente, utilizando las vías, las formas, las relaciones intergrupales, los escenarios y medios del proceso. De esta forma el movimiento del proceso de formación didácticamente está determinado por las relaciones causales que existen entre los componentes y de ellos con la sociedad, la cual determina la esencia de estas relaciones, para poder desarrollar un proceso formativo que responda al encargo social.

La estrategia didáctica que se propone asume de la didáctica sus leyes, principios, componentes, naturaleza, dimensiones y cualidades, las cuales quedan explicitadas en las diferentes etapas y pasos que la conforman y están presentes en el proceso de aprendizaje de una segunda lengua. El principio didáctico de la relación dialéctica entre teoría y práctica es fundamental en la dependencia que tiene el aprendizaje de una segunda lengua y en la necesidad que tiene ésta de que sus hombres no se apropien solamente de un sistema de conocimientos, sino que puedan aplicarlos para resolver las demandas sociales.

El profesor tiene que vincular su mensaje educativo con la vida, pues de lo contrario, éste le llegará vacío, abstracto, carente de significación al estudiante y por tanto no se implicará en la tarea de aprendizaje; deberá aprovechar el aprendizaje vivencial de sus estudiantes, apoyarse en este para futuros aprendizajes; impedir por todos los medios el divorcio entre la teoría y la práctica, el discurso donde se absolutice lo teórico y no se tenga a la práctica como el punto inicial para la elaboración de nuevas teorías.

La enseñanza debe ser activa y garantizar un aprendizaje activo, lo cual implica contar con el alumno, con su vida, situarlo como protagonista fundamental del proceso pedagógico. La unidad que debe existir entre lo temático- técnico (objetivo, contenido, método, medio, evaluación) y lo dinámico (relaciones profesor- alumno, alumno-alumno, alumno- grupo, las relaciones que se dan en el proceso), no serán adecuadas si no se vincula la enseñanza con la vida, con la profesión y con el medio social.

La estrategia empleada se divide por etapas y presenta la estructura siguiente:

1. Primera etapa: se realizó al iniciar el semestre de clases regulares con los estudiantes, es una actividad del docente que presenta las siguientes acciones:

a. Diagnóstico: de esta manera se puede conocer cuáles son los conocimientos previos que poseen los estudiantes tanto en Inglés como en el uso de entornos virtuales y las características de estos jóvenes universitarios.

b. Revisión y análisis de documentos: entre ellos el sílabo, los libros de textos que utilizaran los estudiantes, el entorno virtual que se utilizará en el proceso, el aula virtual donde interactuarán estudiantes-docentes-contenidos.

c. Definición de los núcleos temáticos y contenidos: para las actividades que reforzaran los mismos en el transcurso del semestre.

2. Segunda etapa: en ella se desarrolla el proceso de enseñanza-aprendizaje a lo largo del semestre, sus acciones son:

a. Organización del trabajo a través de diferentes niveles estructurales.

b. Caracterización de los módulos de Inglés a impartir por el docente. 


\section{Tercera etapa.}

a. Evaluación: la cual puede ser diagnóstica, sumativa o de procesos. Cabe destacar que aunque se encuentra al final, la misma se efectúa en todas las etapas de la estrategia.

Los informantes claves en total sumaron 114 estudiantes y un docente de Inglés, se analizaron 4 cursos con 2 aulas virtuales, y el período del estudio fue en el primer semestre del 2014, que comprende de mayo a septiembre 2014. En la tabla 1 se detallan las actividades que se le indicaron a los estudiantes para interactuar con el entorno virtual.

Tabla1. Descripción de las actividades realizadas en los módulos de Inglés

\begin{tabular}{|c|c|c|}
\hline Módulo & $\begin{array}{c}\text { Cantidad } \\
\text { de } \\
\text { estudian- } \\
\text { tes }\end{array}$ & Descripción de actividades personales que debe realizar el estudiante \\
\hline $\begin{array}{l}\text { Primero de } \\
\text { Terapia } \\
\text { Respiratoria } \\
\text { Primero de } \\
\text { Nutrición } \\
\text { Primero de } \\
\text { Ingeniería } \\
\text { en Sistemas }\end{array}$ & 32 & $\begin{array}{l}\text { Los alumnos deben descargar o imprimir el sílabo de la asignatura disponible en } \\
\text { el aula virtual. Cabe destacar que el aula virtual posee un resumen sintetizado de } \\
\text { su sílabo ya que tiene especificado el cronograma de las unidades a estudiar en el } \\
\text { módulo. } \\
\text { Los estudiantes pueden, por medio, de la lectura ampliar o enriquecer sus conoci- } \\
\text { mientos o cultura general aunque los contenidos a leer son en L1 puesto que sería } \\
\text { imposible que un alumno de este nivel comprenda totalmente un contenido de esta } \\
\text { categoría en L2. A su disposición pueden consultar en el aula virtual enlaces que les } \\
\text { muestran amplia información de los países angloparlantes más conocidos como el } \\
\text { Reino Unido de Gran Bretaña e Irlanda del Norte y los Estados Unidos de América, } \\
\text { ya que el libro menciona a estos países de primer mundo en términos generales. } \\
\text { Los estudiantes tienen imágenes importantes a observar que no las encontrarán en } \\
\text { el texto utilizado durante el periodo de la asignatura de inglés, por ejemplo Penélo- } \\
\text { pe Cruz a quien en la unidad dos el libro hace referencia pero no muestra ninguna } \\
\text { imagen completa de ella. De esta forma se comprueba el uso del aula virtual como } \\
\text { herramienta de apoyo en los procesos de enseñanza y aprendizaje. } \\
\text { Los alumnos tienen la oportunidad de practicar Listening, destreza receptiva, por } \\
\text { medio de videos. Estas actividades audiovisuales se las realiza una vez en el salón } \\
\text { de clases, sin embargo, el estudiante puede repetirlas desde donde desee las veces } \\
\text { que lo considere conveniente para su total comprensión o interés. Los videos son } \\
\text { motivantes, de corta duración y acordes al tema que se trata dentro de la unidad en } \\
\text { estudio. Por consiguiente, existe comunión entre el texto utilizado y la tecnología a } \\
\text { través del aula virtual. } \\
\text { Los alumnos tienen que empezar a escribir en el salón de clases sobre su perfil per- } \\
\text { sonal, el trabajo comienza en el aula con la guía del docente pero se culmina y per- } \\
\text { fecciona fuera de ella para luego subirlo al aula virtual. De esta manera se conecta la } \\
\text { destreza productiva de writing con el entorno virtual de aprendizaje. }\end{array}$ \\
\hline $\begin{array}{l}\text { Segundo de } \\
\text { Ingeniería } \\
\text { en Sistemas }\end{array}$ & 15 & $\begin{array}{l}\text { Los alumnos deben descargar o imprimir el sílabo de la asignatura disponible en el } \\
\text { aula virtual. Cabe recalcar que el entorno virtual posee un resumen en síntesis de su } \\
\text { sílabo puesto que tiene especificado el cronograma de las unidades a estudiar en el } \\
\text { módulo. } \\
\text { Los estudiantes tienen imágenes importantes a observar que no las encontrarán en } \\
\text { el texto utilizado durante el periodo de la asignatura de inglés, por ejemplo la carrete- } \\
\text { ra boliviana Yungas en dirección norte y sur. De esta forma se comprueba el uso del } \\
\text { aula virtual como herramienta de apoyo en los procesos de enseñanza y aprendizaje. } \\
\text { Los alumnos tienen que empezar a hacer en el salón de clases una composición } \\
\text { sobre una noche inolvidable para ellos, el trabajo comienza en el aula con la guía del } \\
\text { docente pero se culmina y perfecciona fuera de ella para luego subirlo al aula virtual. } \\
\text { De esta manera se conecta la destreza productiva de writing con el entorno virtual } \\
\text { de aprendizaje. } \\
\text { Los alumnos tienen la oportunidad de practicar Listening, destreza receptiva, por } \\
\text { medio de videos. Estas actividades audiovisuales se realizan una vez en el salón de } \\
\text { clases, sin embargo, el estudiante puede repetirlas desde donde lo considere conve- } \\
\text { niente las veces que desee para su total comprensión o interés. } \\
\text { Los videos son motivantes, de corta duración y acordes al tema que se trata dentro } \\
\text { de la unidad respectiva. Como ya se mencionó, existe comunión entre el texto utili- } \\
\text { zado y la tecnología a través del entorno virtual de aprendizaje. } \\
\text { Finalmente, los estudiantes pueden por medio de la lectura ampliar o enriquecer sus } \\
\text { conocimientos o cultura general aunque las lecturas son en español puesto que sería } \\
\text { imposible que un alumno de este nivel comprenda totalmente un contenido de este } \\
\text { tipo en inglés. A su disposición pueden consultar enlaces que los llevan a conocer } \\
\text { un poco más de literatura conectada con el cine, por ejemplo, el libro menciona la } \\
\text { película El Señor de los Anillos, el aula virtual le proporciona información de la misma } \\
\text { a nivel literario, el texto hace referencia a la película Eclipse, el aula virtual le muestra } \\
\text { o recuerda al alumno el tráiler, es oportuno mencionar que el resultado de esto es } \\
\text { una motivación total del estudiante en su clase de inglés. }\end{array}$ \\
\hline
\end{tabular}

El estudiante de esta manera interactúa en la plataforma educativa generando los aprendizajes a su propio ritmo. La docente durante el semestre realizó la observación con un registro diario de campo y 
al culminar el tiempo de la investigación los estudiantes manifestaron la motivación hacia el estudio de la segunda lengua a través de las TIC con las herramientas digitales que se emplearon.

\section{Resultados y Conclusiones}

Las autoras confirman que se deben establecer estrategias en el aula, para enfrentar los retos de enseñar un idioma diferente. En este caso de estudio, se aprovecharon los entornos virtuales en el aprendizaje del inglés como segunda lengua, a través de las aulas virtuales desarrolladas por la UNEMI.

Las autoras afirman que, con el uso de las herramientas TIC a través de un entorno virtual, se presentan mayores oportunidades en el aprendizaje de una segunda lengua, ya que el docente y alumno pueden estar separados con respecto a la sincronía y esto no es obstáculo para que el aprendizaje se lleve a cabo con éxito, esta herramienta de apoyo ayuda a sellar el conocimiento adquirido, para luego perfeccionarlo con la práctica. Por último, como la tecnología permite el aprendizaje de una segunda lengua con mayor rapidez, lo más probable es que el estudiante sienta la suficiente motivación como para desear aprender otros idiomas extranjeros, lo cual lo transformará social y culturalmente, preparándolo para cruzar las fronteras.

\section{Referencias}

1. Alexander, R.Culture and Pedagogy. International Comparisons in Primary Education. Oxford: Blackwell Publishers Ltd. 2000.

2. Alexander, R.Towards Dialogic Teaching. Rethinking classroom talk. Thirsk, North Yorkshire: Dialogs.2004.

3. Barboza Norbis, L."Informe sobre la organización curricular del dominio lingüístico delos Programas y Planes de Estudio de ANEP desde 1941 a 2007" en ANEP. CODICEN. COMISIÓN DE POLIITICAS LINGÜÍSTICAS EN LA EDUCACIÓN PÚBLICA: Documentos e Informes Técnicos de la Comisión de Políticas Lingüísticas en la Educación Pública. Diciembre de 2006 - Noviembre de 2007. En línea: http://www.oei. es/pdfs/informe_linguistica_uruguay.pdf

4. Behares, Le, Brovetto, C. "Políticas lingüísticas en el Uruguay. Análisis de sus modos de establecimiento" en C. Brovetto (comp.): Primer Foro Nacional de Lenguas de ANEP, pp. 143-174. Montevideo: ANEP. CODICEN. (2009a)
5. Behares, Le, Brovetto, C."Sobre las referencias al dominio lingüístico en las leyes de educación de Uruguay" en Encontro Internacional de pesquisadores de políticas lingüísticas 4, pp. 95-102. Santa Maria: Associação de Universidades Grupo Montevideo / Universidad Federal de Santa María. (2009b)

6. Brovetto, C. "Educación bilingüe de frontera y políticas lingüísticas en Uruguay" en Pro- Posições. Dossiê Educaçãofronteiriça Brasil/Uruguay, línguas e sujeitos, vol. 21, No 3 (Sept./Dec. 2010), pp. 25-43. Campinas: Faculdade de Educação, Unicamp. En línea: http://www.scielo.br/scielo. php?pid=S0103- 73072010000300003\&script=sci_arttext

7. Cristal, D. The Cambrigde encyclopedia of the English language. Cambrigde. CUP. 1995.

8. Corina, J. y Dirnestein, P. 2009. Diseño curricular y nuevas generaciones. Editorial Virtual Argentina.

9. Fuertes, P. y Samaniego, E. El Inglés con fines específicos: Rasgos distintivos). Documento en línea. Disponible en [http://www.pedrofuertes.net/ uploads/ESP.pdf]. Consultadoen: [20 de agosto de 2014]

10. Hayes, D."Planning for success: Culture, engagement and power in English language education innovation" en C. Tribble (ed.): Managing change in English language teaching: Lessons from experience, pp. 47-60. London: British Council. Enlínea: http://www.teachingenglish.org.uk/ sites/teacheng/ files/B330\%20MC\%20in\%20 ELT\%20book_v7.pdf. 2012

11. Levy, M.Computer-Assisted Language Learning: Context and Conceptualization. Oxford: Oxford University Press. 1997.

12. Ley No 18.437. Ley General de Educación". Montevideo, 12 de diciembre de 2008. En línea: http://www.parlamento.gub.uy/ leyes/AccesoTextoLey.asp?Ley=18437\&Anchor=

13. Murray, D, Christison, Ma."Understanding innovation in English language education: Contexts and issues" en C. Tribble (ed.): Managing change in English language teaching: Lessons from experience, pp. 61-74. London: British Council. Enlínea: http://www.teachingenglish.org.uk/ sites/ teacheng/files/B330\%20MC\%20in\%20ELT\%20 book_v7.pdf. 2012.

14. Nicolás, B. Las estrategias de aprendizaje en idioma inglés. 
15. Orozco, J. El Docente y el Aula. Universidad Estatal de Milagro. 2011

16. Rubio, M. Factores que intervienen en el aprendizaje de una segunda lengua. Revista digital innovación y experiencias educativas [en línea]. 21 de noviembre 2011, $n^{\circ} 46$. Consultado en: [20 de octubre de 2014]. Disponible en [http:// www.csi-f.es/archivos/andalucia/ensenanza/revistas/iee/Numero_46/MARIA_DEL_CARMEN_ RUBIO_OSUNA_02.pdf].

17. Salaberry, Mr. "The Use of Technology for Second Language Learning and Teaching: A Retrospective" en The Modern Language Journal, vol. 85, No 1, Special Issue: A Century of Language Teaching and Research: Looking Back and Looking Ahead, Part 2 (Spring), pp. 39-56. 2001

18. Spada, N."Communicative Language Teaching: Current Status and Future Prospects" en J. Cummins; C. Davison (eds.): International Handbook of English Language Teaching, Part 1, pp. 271-288. New York: Springer Science and Business Media. Serie Springer International Handbooks of Education 15. 2007.

19. Stanley, G. Language Learning with Technology. Ideas for integrating technology in the classroom. Cambridge: Cambridge University Press.2013

20. Stoller, F. "Innovation as the hallmark of effective leadership" en M. A. Christison; D. Murray (eds.): Leadership in English language education: Theoretical foundations and practical skills for changing times, pp. 73-84. New York: Routledge.2009

21. Stoller, F. "Catalyst for innovation" en M. A. Christison; F. L. Stoller (eds.): A handbook for language program administrators (Second edition). Miami: Alta Book Center. 2012.

22. Tribble, C. Managingchangein English language teaching: Lessons from experience. London: British Council. 2012. Enlínea: http:// www. teachingenglish.org.uk/sites/teacheng/files/ B330\%20MC\%20in\%20ELT\%20book_v7.pdf

23. Valenzuela, J. Lingüística contrastiva inglés-español: Una visión general. Documento en línea. Disponible en [http://www.um.es/lincoing/ jv/2002\%20Contrastivo\%20Carabela.pdf]. Consultadoen: [20 de septiembre de 2014]

24. Cambridge: MIT Press.

25. Vygotsky, LS. Thought and Language. Cambridge: MIT Press.1986
26. Wells, G. Dialogic Inquiry: Towards a Sociocultural Practice and Theory of Education. Cambridge: Cambridge University Press. 1999 